\title{
O Impacto das Atividades do Grupo PET no Aprimoramento de Soft Skills Requeridos pelo Mercado de Computação do Maranhão: Uma Análise da Visão dos Discentes
}

\author{
Arthur Passos $^{1}$, André Barreto ${ }^{1}$, Brenno Nascimento ${ }^{1}$, Felipe Silva ${ }^{1}$, \\ Gabriel Costa $^{1}$, Yandson Costa ${ }^{1}$, Davi Viana ${ }^{2}$, Luis Rivero ${ }^{1,2}$ \\ ${ }^{1}$ Programa de Educação Tutorial do Curso de Ciência da Computação (PETComp) \\ Universidade Federal do Maranhão (DEINF/UFMA) - São Luís - MA - Brasil \\ ${ }^{2}$ Programa de Pós-Graduação em Ciência da Computação (PPGCC), \\ Universidade Federal do Maranhão (UFMA) - São Luís, MA - Brasil \\ \{arthur.passos, barreto.andre, brenno.izaias, felipe.henrique, \\ gabriel.jesus, yandson.jesus\}@discente.ufma.br, \\ $\{$ davi.viana, luis.rivero\}@ufma.br
}

\begin{abstract}
The computer practitioner needs to acquire the most varied skills to remain competitive in the job market. Among these skills, the so-called soft skills (or non-technical skills) are personal attributes that allow the individual to perform a good interaction with the world around him. In such context, the Tutorial Educational Program (PET) appears as an alternative to develop such skills during higher education. However, there are few analyzes on how a student can develop soft skills while carrying out activities within the scope of PET. In this paper, through a form, the students' perception about the impact of the activities carried out in PET for the development of soft skills was obtained, considering the context of the state of Maranhão. The results point to the positive impact of the program's activities for this purpose, in addition to identifying opportunities for improvement.
\end{abstract}

Resumo. O profissional de computação precisa adquirir as mais variadas habilidades para se manter competitivo no mercado de trabalho. Entre essas habilidades, as denominadas soft skills (ou habilidades não-técnicas) são atributos pessoais que permitem ao indivíduo desempenhar uma boa interação com o mundo ao seu redor. Nesse contexto, o Programa de Educação Tutorial (PET) surge como uma alternativa para desenvolver tais habilidades durante o ensino superior. No entanto, existem poucas análises sobre como um discente pode desenvolver soft skills durante a realização de atividades no âmbito do PET. Neste artigo, através de um formulário, foi obtida a percepção dos discentes sobre o impacto das atividades realizadas no PET para o desenvolvimento de soft skills, considerando o contexto do estado do Maranhão. Os resultados apontam para o impacto positivo das atividades do programa para este fim, além de identificar oportunidades de melhoria.

\section{Introdução}

Com a constante mudança nos processos do trabalho, há uma necessidade de implementar comportamentos que melhorem o potencial de cada indivíduo. Nesse contexto, o mercado 
de trabalho e a academia sugerem que um profissional qualificado deve possuir habilidades além das técnicas para a realização das suas tarefas [Ahmed et al. 2012]. Desta forma, é necessário que o profissional tenha habilidades intrapessoais e interpessoais, ou habilidades não-técnicas (soft skills), que o permitam exercer a sua função de forma apurada.

As soft skills podem ser classificadas como traços de personalidade, objetivos, motivações e preferências que são valorizadas pelo mercado de trabalho, na escola ou em outros contextos [Heckman and Kautz 2012]. Esse tipo de habilidades, conhecimentos e atitudes são esperados de um graduando que deseja adentrar ao mercado de trabalho e obter sucesso profissional [Hillage and Pollard 1998]. No entanto, apesar da sua importância, há relatos de discentes sobre a falta de preparo na aquisição e desenvolvimento de soft skills, por falta de interesse por parte dos discentes e/ou falta de atividades de ensino que visem prepará-los no desenvolvimento das mesmas [Succi and Canovi 2020].

Considerando o conjunto de oportunidades que a universidade oferece aos discentes para auxiliar na sua formação e capacitação, o Programa de Educação Tutorial (PET) tem se tornado um destaque devido à sua abrangência e potencial formador [Andrade et al. 2020]. Segundo o Ministério da Educação (MEC) ${ }^{1}$, o PET é um grupo formado por discentes com tutoria de um docente que tem por base o tripé da universidade: ensino, pesquisa e extensão. Esse programa viabiliza o desenvolvimento de atividades extracurriculares, ajudando na formação acadêmica dos envolvidos, e melhorando a qualidade acadêmica dos cursos de graduação a que ele está direcionado. Segundo Leite et al. (2019), essas atividades extracurriculares contribuem significativamente para a formação de indivíduos que estejam preocupados com o trabalho em equipe, a proatividade, a análise de problemas, além do compromisso com objetivos de apoiar a sociedade.

Considerando o contexto acima, Instituições de Ensino Superior com Grupos PET na área de computação, podem auxiliar os futuros profissionais no desenvolvimento de soft skills, uma vez que a indústria exige cada vez mais estes atributos como pré-requisito de seleção [França and Mellet 2016]. Nesse contexto, este trabalho visa analisar quais e como as atividades desenvolvidas pelo grupo PET de Computação (PETComp) da Universidade Federal do Maranhão (UFMA) têm contribuído para o desenvolvimento de habilidades não-técnicas por parte dos discentes. Para isso, foi feito um levantamento das soft skills mais requisitadas pelo mercado de Tecnologia da Informação (TI), considerando vagas de emprego agregadas no trabalho de Costa et al. (2020). Com os dados do levantamento, as diversas atividades realizadas pelo grupo PETComp foram avaliadas a partir de um questionário do ponto de vista dos discentes, com relação ao seu potencial para permitir o desenvolvimento destas soft skills. Finalmente, sugestões de melhoria para tornar as atividades mais aderentes ao processo de formação foram indicadas pelos discentes e analisadas pelos autores deste trabalho. Com estes resultados, outros grupos PET na área de computação podem analisar que atividades podem ser replicadas nos seus respectivos grupos para incentivar o desenvolvimento de soft skills.

Além dessa seção introdutória, este artigo está organizado da seguinte forma. A Seção 2 apresenta os trabalhos relacionados a este trabalho. A Seção 3 descreve a metodologia de pesquisa utilizada. Os resultados obtidos são analisados e discutidos na Seção 4. Por fim, as conclusões e os trabalhos futuros são apresentados na Seção 5.

\footnotetext{
${ }^{1}$ http://portal.mec.gov.br/pet
} 


\section{Trabalhos Relacionados}

Como França e Mellet (2016) apontam, a busca por profissionais dotados de habilidades não-técnicas é cada vez mais frequente na indústria de TI. Tendo isso em vista, para entender quais soft skills o mercado exige, a análise de ofertas de vagas vem sendo feita. Além disso, como forma de contribuir no desenvolvimento de tais habilidades, as universidades têm mostrado algumas oportunidades para que isso ocorra.

Pedrosa e Ferreira da Silva (2019) identificaram as principais soft skills requeridas para vagas de emprego na posição de gerente de projetos na área de Tecnologia da Informação no Brasil, e para isso foi utilizada uma abordagem qualitativa. Os dados sobre as vagas foram coletados através de uma pesquisa na plataforma Linkedin, e então, foram analisados os requisitos solicitados aos candidatos em cada uma delas, a fim de identificar as soft skills requeridas. Essas ocorrências de soft skills foram contabilizadas, gerando como resultado um ranking das soft skills baseado na frequência em que elas apareciam nas vagas de emprego. Ao todo, os autores identificaram 43 habilidades, entre elas: comunicação, liderança, negociação e relacionamento interpessoal.

Por sua vez, Matturro et al. (2019) realizaram uma revisão sistemática identificando quais soft skills são relevantes para a prática da engenharia de software. Após a conclusão da revisão da literatura, 44 artigos relatando 30 categorias de soft skills foram identificados. Estes artigos relataram ao todo, 30 categorias de soft skills, considerando habilidades de comunicação, trabalho em equipe, análise, organização e interpessoais. Segundo os autores, as soft skills são normalmente identificadas a partir da aplicação de questionários e análise de vagas de emprego.

Em outro trabalho, González-Morales et al. (2011) reportaram a tentativa de ensinar soft skills a partir de atividades práticas no contexto de uma disciplina de engenharia de software. Para isso, projetos reais foram executados, em que grupos de discentes realizavam atividades de supervisão, gerenciamento de projetos, garantia da qualidade e tomada de decisões. Os projetos tinham o objetivo de trabalhar soft skills como: busca e classificação de informações, escrita de relatórios, trabalho em equipe, liderança e supervisão, tomada de decisões, gerenciamento de conflitos, pensamento crítico, avaliação e comunicação de resultados. Os autores indicaram que, ao analisar o desempenho dos discentes, foi possível observar a aquisição destes soft skills e que ter atividades definidas durante o projeto pode auxiliar no desenvolvimento destas habilidades.

Os trabalhos acima apontam para o interesse da comunidade acadêmica para o desenvolvimento de soft skills durante a formação superior dos discentes. Embora GonzálezMorales et al. (2011) tenham realizado um projeto composto de diversas atividades visando auxiliar na obtenção de soft skills, as atividades extra-curriculares de programas como o PET devem ser avaliadas para auxiliar na escolha das mesmas dependendo do objetivo formador dos discentes. Além disso, apesar dos estudos abrangentes identificando os soft skills requisitados no mercado de trabalho nacional e internacional, um olhar focado em locais específicos como cidades e estados, pode auxiliar os discentes a entenderem como está seu preparativo para ocupar vagas mais próximas após sua saída na universidade ou durante a procura de um estágio. Este trabalho propõe apresentar estas informações para que outros grupos PET possam priorizar atividades dependendo do objetivo formador dos discentes, e permitir entender o que pode ser melhorado para aumentar o impacto das atividades no desenvolvimento de soft skills. 


\section{Metodologia}

Este trabalho foi realizado em duas etapas: (1) identificação das soft skills mais solicitadas em vagas de emprego do estado do Maranhão; e (2) análise das atividades realizadas pelo PETComp UFMA com base na sua contribuição para o desenvolvimento dessas soft skills. O processo aplicado para a pesquisa é explicado nas subseções a seguir.

\subsection{Coleta e Análise de soft skills do Estado do Maranhão}

Para definir o conjunto de soft skills que são solicitados nas vagas de Tecnologia da Informação no estado do Maranhão, foram reaproveitados os dados fornecidos por Costa et al. (2020). Embora esse trabalho foque na identificação dos principais conteúdos de Engenharia de Software que são solicitados pela indústria, a base de dados inicial possui vagas de emprego que são voltadas para profissionais da área de computação de um modo geral. Esta base possui um total de 72 vagas de emprego disponibilizadas durante o primeiro semestre de 2020 em sites de recrutamento (LinkedIn, Trabalha Brasil, Info Jobs e Catho), editais de concursos públicos do estado e grupos de WhatsApp e Facebook.

Com posse dos dados das vagas de emprego, foi feita uma análise das suas descrições, especificamente das habilidades, conhecimentos e competências requeridas, visando identificar as soft skills solicitadas pelo mercado de TI no Maranhão. Para classificar as soft skills identificadas, foram utilizados como base os trabalhos de Schulz (2008), Ferreira da Silva (2019) e Matturro et al. (2019). Vale ressaltar que o trabalho de Schulz (2008) foi escolhido, pois foca em soft skills importantes do ponto de vista acadêmico, enquanto os outros, analisam soft skills advindos de revisões da literatura ou a opinião de profissionais. Posteriormente, foi feita uma contagem de ocorrências de cada uma das habilidades encontradas nas descrições das vagas, organizando-as em um ranking.

\subsection{Formulário de Avaliação de Atividades}

Uma vez obtido o ranking de soft skills advindo da análise de vagas de emprego do estado do Maranhão, foram organizadas as atividades realizadas pelo PETComp no período de Janeiro de 2018 a Março de 2021. O objetivo era apresentar aos participantes (membros atuais e ex-membros do grupo PETComp) atividades que de uma ou outra forma eles poderiam ter realizado. A partir deste levantamento, 12 atividades foram identificadas e foi elaborado um formulário ${ }^{1}$ através da plataforma Google Forms. Este formulário, listava as atividades e solicitava que os discentes informassem quais soft skills eles acreditavam ter alcançados com as mesmas. Além disso, os discentes podiam informar em quais atividades haveria oportunidades de melhoria para aumentar o seu impacto no desenvolvimento de soft skills. Outras perguntas foram feitas para que os discentes pudessem citar outras atividades do grupo que tiveram impacto positivo na sua formação, e dar sugestões para o melhor desempenho e aproveitamento das atividades.

A seguir, são apresentadas os resultados da análise de vagas de emprego visando identificar soft skills requeridas no estado do Maranhão. Além disso, são apresentadas as atividades desenvolvidas pelo grupo PETComp, explicando seu objetivo e a avaliação dos discentes com relação ao seu impacto para o desenvolvimento das soft skills identificadas.

\footnotetext{
${ }^{1}$ https://drive.google.com/file/d/10VTodLqCa4rG5TfYGpILrbW6gsK9rcoA/view?usp=sharing
} 


\section{Resultados e Discussão}

Após a análise de 72 vagas de emprego, foram identificadas 13 soft skills, ordenadas por número de ocorrências. A Figura 1 apresenta o ranking dos soft skills no estado do Maranhão utilizando a base de dados de Costa et al. (2020). As soft skills identificadas são: Comunicação, Criatividade, Trabalho em Equipe, Pensamento Crítico, Organização, Responsabilidade, Fluência em Inglês, Proatividade, Liderança, Autonomia, Agilidade, Empatia e Interpretação de Texto. A ocorrência máxima foi de 12 para a habilidade comunicação, enquanto as habilidades Agilidade, Empatia e Interpretação de Texto, foram as menos citadas, com 2 citações cada. Vale ressaltar que embora outras soft skills tenham sido citadas em outros trabalhos, neste artigo foram consideradas no questionário apenas as levantadas pela análise de vagas de emprego descrita na Seção 3.1.

Ocorrência de Soft Skills em Vagas de Emprego Identificadas por Costa et al. (2020)

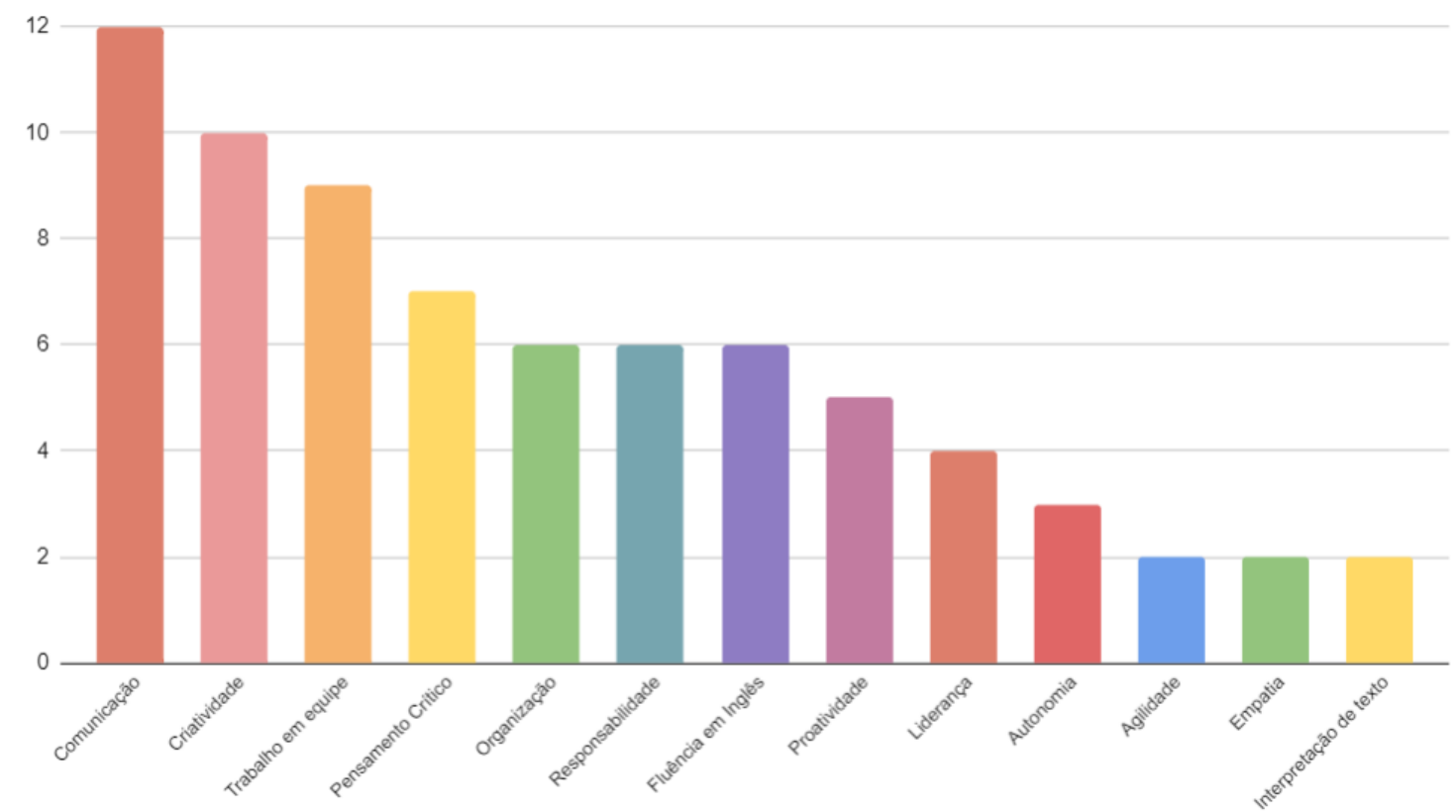

Figura 1. Ranking de soft skills do mercado de TI no Maranhão

Com relação às atividades do PETComp, foram selecionadas as 12 principais atividades desenvolvidas ao longo do período de 2018 a 2020, sendo elas: Recepção de Calouros, que visa aproximar discentes ingressantes da universidade e do curso; Acompanhamento de Discentes, que visa ajudar discentes com monitorias em algumas disciplinas do curso; Fábrica de Software, que é responsável pelo desenvolvimento de sistemas necessários como sites e aplicativos dentro da Universidade e para a comunidade local; Pesquisador Aprendiz, que objetiva inserir os integrantes em grupos de pesquisa e desenvolver projetos; Minicursos e Oficinas, que visa compartilhar conhecimentos com a comunidade acadêmica através do desenvolvimento de minicursos e oficinas; CinePET, atividade de extensão que consiste na produção de conteúdos audiovisuais sobre aspectos atuais relacionados ao mercado de trabalho e pesquisas/acontecimentos na área de tecnologia da informação; Eventos de Computação, que tem como objetivo promover e organizar eventos na área da computação; Participação dos Eventos do PET, que visa 
incentivar a participação e organização em eventos promovidos e organizados pelos grupos PET; Expressão Escrita, que representa a escrita de artigos dentro do grupo PET, sendo fortemente incentivada a participação e apresentação de tais artigos em eventos; Biblioteca PETComp, que visa promover o ensino ao divulgar material bibliográfico ou digital produzido para a comunidade acadêmica; Divulgação do PETComp, que visa divulgar e difundir, através das mídias sociais do grupo, as atividades e materiais desenvolvidos; e Apoio à Escola, que visa desenvolver maneiras de colaborar com escolas de Ensino Fundamental e Médio no ensino de conceitos de computação, além de atrair meninas para as Ciências Exatas, em especial à Computação. Informações sobre como estas atividades têm sido realizadas ao longo dos anos no âmbito do grupo PET podem ser encontradas em trabalhos anteriores [Ferreira et al. 2015, Leite et al. 2019].

Ao todo, 23 discentes membros atuais e ex-membros do grupo PETComp responderam ao questionário. Dessas 23 pessoas, 11 (48\%) delas estão presentes atualmente no ano de 2021, 20 (87\%) estiveram presentes em 2020, 11 (48\%) em 2019, 7 (30\%) em 2018, 2 (8\%) em 2017 e 1 (4\%) anterior a 2017, sendo que muitas delas permaneceram no Programa por mais de um ano. Do conjunto de pessoas que permaneceram no grupo por mais de um ano, 7 (30\%) delas permaneceram no PETComp por no máximo por 3 anos, enquanto $11(48 \%)$ delas permaneceram por no máximo 2 anos e 5 (22\%) delas permaneceram por no máximo 1 ano. Embora houve respondentes fora do intervalo especificado de atividades (2018 e 2020), uma vez que as atividades se repetem ao longo dos anos (antes mesmo do limite inferior de 2018), as respostas destes discentes foram consideradas. Além disso, todos os discentes que responderam ao questionário participaram da realização de pelo menos 5 e até no máximo 12 atividades. Vale ressaltar que caso um discente não tenha participado de uma atividade, ele o informou no formulário.

Na Tabela 1 são apresentados os dados quantitativos das respostas dos discentes que participaram das atividades desenvolvidas dentro do PETComp sobre quais soft skills eles acreditam que essas atividades ajudaram a desenvolver. Ao responder o questionário, o discente informou quais soft skills cada atividade ajudou a desenvolver e estas ocorrências foram contabilizadas. Ao final da contagem, as atividades foram ordenadas de maior a menor pela quantidade de vezes que elas foram relacionadas a um soft skill, enquanto os soft skills foram ordenados de maior a menor pela quantidade de vezes que estas foram citadas em alguma atividade. Ao analisar a tabela, nota-se que as três primeiras atividade que mais contribuíram para o desenvolvimento de soft skills segundo os discentes foram: Eventos de Computação, Expressão Escrita e Fábrica de Software. Por sua vez, as soft skills mais desenvolvidas ao longo das atividades realizadas no PET foram, segundo os discentes: Organização, Responsabilidade e Comunicação.

Com relação às perguntas de sugestão para cada atividade, os relatos dos discentes apresentam ideias de melhorias que podem ser implantadas em execuções futuras das mesmas para que estas possam ser melhor aproveitadas no desenvolvimento e/ou aprimoramento de diferentes soft skills. A seguir, são apresentados alguns trechos das respostas mais detalhadas com relação às atividades avaliadas.

“Nas atividades de eventos, ... É preciso, se colocar a frente de pontos que, por vezes, são esquecidos e se pronunciando quando algo parece estar errado. Assim, fazendo com que a soft skill de liderança floresça com mais facilidade.” - Participante P05 sobre a Atividade Eventos de Computação 
Tabela 1. Avaliação das soft skills adquiridas ao participar das atividades realizadas no PETComp.

\begin{tabular}{|c|c|c|c|c|c|c|c|c|c|c|c|}
\hline Atividade / Soft Skill & 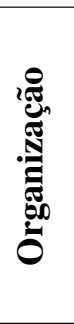 & 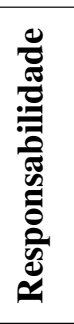 & 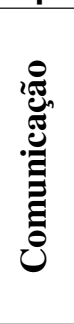 & 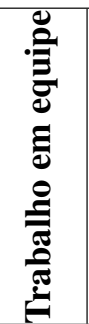 & 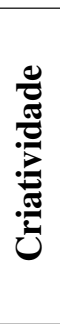 & 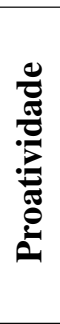 & 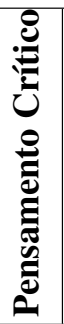 & 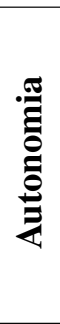 & 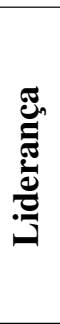 & 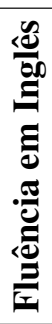 & 胥 \\
\hline Eventos de Computação & 17 & 18 & 19 & 16 & 10 & 16 & 5 & 12 & 5 & 2 & 120 \\
\hline Expressão Escrita & 17 & 15 & 11 & 13 & 12 & 9 & 18 & 11 & 7 & 4 & 117 \\
\hline Fábrica de Software & 16 & 14 & 10 & 15 & 14 & 10 & 9 & 8 & 8 & 4 & 108 \\
\hline Recepção dos Calouros & 18 & 16 & 17 & 19 & 11 & 13 & 1 & 6 & 6 & 0 & 107 \\
\hline Minicursos e Oficinas & 14 & 12 & 15 & 13 & 13 & 8 & 5 & 8 & 5 & 0 & 93 \\
\hline Acompanhamento de Discentes & 12 & 15 & 11 & 10 & 5 & 10 & 8 & 7 & 6 & 1 & 85 \\
\hline Participação dos Eventos do PET & 12 & 12 & 17 & 11 & 3 & 8 & 9 & 5 & 3 & 1 & 81 \\
\hline Pesquisador Aprendiz & 11 & 12 & 8 & 3 & 5 & 9 & 13 & 10 & 2 & 4 & 77 \\
\hline Divulgação do PETComp & 3 & 7 & 8 & 5 & 9 & 3 & 3 & 3 & 3 & 0 & 44 \\
\hline Biblioteca PETComp & 8 & 6 & 4 & 6 & 6 & 3 & 4 & 0 & 3 & 1 & 41 \\
\hline Apoio a Escola & 3 & 4 & 4 & 3 & 5 & 3 & 0 & 1 & 1 & 0 & 24 \\
\hline CinePET & 4 & 4 & 2 & 3 & 1 & 0 & 1 & 1 & 1 & 0 & 17 \\
\hline Total & 132 & 131 & 122 & 114 & 89 & 89 & 76 & 71 & 49 & 17 & \\
\hline
\end{tabular}

"A execução das atividades deveria ser mais voltada à expressão escrita, para que, quando chegar o momento de escrever os relatos, já se tenha material e referências e um acompanhamento mais adequado dos escritores mais novos pelos mais experientes. - Participante P03 sobre a Atividade Expressão Escrita

"Sugiro orientação em engenharia de software, técnicas como scrum e o básico de IHC. Períodos de qualificação, seguidos de intensa produção e validação por parte dos stakeholders. - P10 sobre a Atividade fábrica de software

"Acredito que organização e responsabilidade é o que mais falta nessa atividade que muitas vezes é feita informalmente. Estabelecer cronogramas, separação de funções e reuniões semanais com essa equipe deve direcioná-los em tratar isso de forma séria e competente.” - Participante P08 sobre a Atividade de Divulgação do PETComp

"Seria interessante que os trabalhos de pesquisa fossem apresentados a todo o grupo, a fim de desenvolver habilidades de comunicação nos autores, e de pensamento crítico por partes daqueles que estarão assistindo as apresentações e deverão tirar dúvidas e dar suas considerações sobre cada trabalho. Para que haja o desenvolvimento da habilidade de liderança, é interessante que os trabalhos de pesquisa próprios do grupo PET sejam assumidos por integrantes diferentes como primeiros autores." - Participante P22 sobre a Atividade Pesquisador Aprendiz

"Trabalho em equipe e proatividade são muito importantes nessa atividade, sempre mantendo é claro a comunicação, para isso trabalhar com o Trello fazer checklists é o ideal para melhorar a atividade ou até mesmo quadros de desenvolvimento para deixar claro o trabalho dos integrantes do grupo." - Participante P02 sobre a Atividade Minicursos e Oficinas 
Com relação à pergunta "Há alguma(s) outra(s) atividade(s) não listada(s) que contribuiu(iram) para sua formação, em relação ao desenvolvimento de soft skills? Se sim, como esta(s) atividade(s) impactou(aram) na sua formação?”, os discentes relataram como o programa tem contribuído para o seu desenvolvimento. Embora nenhuma atividade nova foi citada, relatos dos discentes focaram principalmente no detalhamento de subatividades realizadas dentro do âmbito das grandes atividades do PETComp listadas na Tabela 1. Segundo os discentes, algumas atividades do PETComp ajudaram a lidar com situações de pressão (Pensamento Crítico), aprender a ouvir e saber se impor quando necessário e ao debater seus argumentos (Comunicação e Autonomia), lidar com pessoas e suas diferenças (Trabalho em Equipe), organização de tempo e materiais (Organização e Responsabilidade), pensar em novas formas de se construir algo (Criatividade). Algumas citações sobre estes pontos são apresentados a seguir.

"A atividade de Criar um PodCast, por enquanto, foi a que causou um impacto evidente mais rápido, pois nele foi testada minha capacidade de liderança, criando algo que eu não tinha experiência alguma e fazendo com que eu testasse minhas habilidades comunicativas de maneira direta sendo Host de alguns episódios.” - Participante P05

“As reuniões me ajudaram a cumprir horários e ouvir àqueles que discordam de mim. Também me ajudou a criar coragem para falar quando minha opinião não é aceita pela maioria. O PET me ajudou a lidar com a pressão.” - Participante P09

“As comissões internas PET Mídias e Patrimônio. Contribuíram imensamente com as habilidades comunicativas e assertivas; e organização, administração e planejamento, respectivamente." - Participante P11

Com relação à pergunta "Quais mudanças (sugestões na organização, novas atividades, treinamento de outros soft skills) você sugeriria na execução das atividades do PETComp para ter um melhor aproveitamento das mesmas?", os discentes relataram suas opiniões sobre mudanças que poderiam ocorrer nas atividades para que elas pudessem ser mais proveitosas. Vários discentes citaram a adição de novas atividades, apontando os benefícios que elas trariam para o desenvolvimento dos petianos, como a criação de dois tipos de podcasts, um focado na área da computação e outro na área acadêmica, apontando que essa atividade ajuda bastante a desenvolver soft skills de pensamento crítico e de comunicação. Outros respondentes sugeriram mudanças na organização das atividades, como uma rotação maior de liderança nas tarefas e um foco maior nas tarefas ativas. Alguns comentários que apontam oportunidades de melhoria são apresentados abaixo.

"Projetos no PETComp tomam iniciativa mas às vezes acabam adormecendo por longos períodos. Acredito que o foco na atividade ativa e no objetivo original dela sejam de estímulo contínuo aos petianos, afinal concluir um projeto é sempre revigorante. Então proponho o planejamento de atividades de forma que as eventuais demandas novas não adormeçam completamente os outros projetos.” - Participante P03

"Acredito que muitas das atividades sofrem com uma falta de organização e responsabilidade por parte dos petianos, o estabelecimento de regras do funcionamento de atividades desde o começo deve ser incentivado para que eles não venham se perder durante a realização de atividades, é importante ressaltar o gerenciamento mútuo entre os petianos, pois a falta de um prejudica o todo." - Participante P08

“Talvez seja interessante convidar outros grupos PET de outros cursos para que 
desenvolvam atividades elou levem discussões fora do contexto da computação para dentro do PETComp. Isso pode ajudar a equipe a desenvolver habilidades mais gerais, como pensamento crítico e comunicação. [...] talvez seja interessante que os integrantes recebam profissionais de computação de diferentes setores de TI [...] para que relatem suas experiências, podendo até relacionar as habilidades necessárias para o desenvolvimento de seus trabalhos.” - Participante P22

“Acredito que dessas soft skills, a menos desenvolvida no PETComp seja a de fluência em inglês, por que ela é desenvolvida só na atividade de Submissão Escrita, e isso só caso você decida pegar algum artigo em inglês, então acho que seria uma boa ideia ter alguma atividade que desenvolva mais isso.” - Participante P07

A partir dos resultados e relatos apresentados acima, outros grupos PET podem avaliar as atividades realizadas pelo PETComp e como podem implementar ou melhorar essas atividades dentro do seu próprio grupo para um melhor desenvolvimento de uma ou mais soft skill pelos seus membros. Além disso, docentes e discentes podem utilizar alguns métodos ou aspectos das atividades na elaboração das disciplinas e desenvolvimento de práticas de estudo para que essas habilidades sejam desenvolvidas por alunos fora do grupo PET.

\section{Conclusão}

O presente trabalho apresentou uma análise de quais são as soft skills solicitadas pelo mercado de trabalho no estado do Maranhão e como o Grupo PETComp da UFMA pode contribuir com o desenvolvimento das mesmas a partir as atividades realizadas nele. Os resultados apontam que, na visão dos discentes, as atividades têm cumprido seu papel em auxiliá-los no seu processo de amadurecimento e crescimento pessoal. Valem ser citadas as atividades de Eventos de Computação, Expressão Escrita, Fábrica de Software e Recepção de Calouros que constam com o maior índice de soft skills desenvolvidas. Além disso, o grupo tende a desenvolver melhor soft skills relacionadas com Organização, Responsabilidade, Comunicação e Trabalho em Equipe (todas com mais de 100 ocorrências durante as atividades realizadas). Ainda assim, há espaço para melhorias de algumas atividades, como incentivar o ato de falar em público juntamente com a interação de petianos com outros indivíduos, saber ouvir feedbacks e aplicar melhorias, como na organização das atividades, a fim de causar menos estresse. Além disso, podem-se planejar as atividades de forma que ocorram na maioria das vezes em equipe, visando assim evitar sobrecarga e, por sua vez, insegurança. Um dos pontos mais comentados pelos discentes, foi a presença dos petianos sempre nas mesmas tarefas, então pretende-se promover uma maior rotação de atividades entre os petianos, a fim de proporcionar o aperfeiçoamento de uma grande variedade de soft skills.

Com este trabalho, foi possível entender a visão dos discentes sobre a importância de se desenvolver as soft skills, pois essas são características imprescindíveis para uma boa relação interpessoal dentro do ambiente das empresas, implicando, assim, no sucesso dos projetos desenvolvidos por esses futuros profissionais. Dessa forma, pretendese continuar trabalhando na capacitação dentro do PET, além de implementar as opiniões dadas pelos integrantes e ex-integrantes nas perguntas individuais, para futuramente avaliar como essas mudanças contribuíram para o refinamento das atividades e se há algum tipo de impacto na formação acadêmica e desenvolvimento profissional dos petianos e 
ex-petianos. O grupo busca o futuro desenvolvimento do trabalho para avaliar detalhadamente o impacto de cada atividade e a forma que cada soft skill é adquirida.

\section{Referências}

Ahmed, F., Capretz, L. F., and Campbell, P. (2012). Evaluating the demand for soft skills in software development. It Professional, 14(1):44-49.

Andrade, J. D. I., da Silva, T. R., Rodrigues, F. L., Saldanha, D. G., Pinto, G. F., and Junior, J. A. S. D. F. (2020). O impacto do programa de educação tutorial no desempenho acadêmico dos seus integrantes. Anais do Salão Internacional de Ensino, Pesquisa e Extensão, 12(2).

Costa, Y., Gomes, D., Santos, S., Pinto, N., Viana, D., Braz, G., and Rivero, L. (2020). Identifying improvement opportunities in software engineering education at the maranhão state: Listening to voices from academy and industry. In Proceedings of the 34th Brazilian Symposium on Software Engineering, pages 860-869.

Ferreira, J., Ribeiro, J., de Freitas Júnior, A., Sousa, G., da Silva, I., Santos, H., Menezes, M., Silva, A., Ferreira, B., de Jesus, L., et al. (2015). Educação tutorial em ciência da computação: uma proposta de sistematização. In Anais do XXIII Workshop sobre Educação em Computação, pages 161-167. SBC.

França, C. and Mellet, D. (2016). Soft skills required! uma análise da demanda por competências não-técnicas de profissionais para a indústria de software e serviços. Anais do IX Fórum de Educação em Engenharia de Software (FEES 2016), pages 101-112.

González-Morales, D., De Antonio, L. M. M., and García, J. L. R. (2011). Teaching "soft" skills in software engineering. In 2011 IEEE Global Engineering Education Conference (EDUCON), pages 630-637. IEEE.

Heckman, J. J. and Kautz, T. (2012). Hard evidence on soft skills. Labour economics, 19(4):451-464.

Hillage, J. and Pollard, E. (1998). Employability: developing a framework for policy analysis. Department for Education and Employment, Suffolk, Inglaterra, 85 edition.

Leite, F., Cardoso, G., Costa, Y., Estrela, I., Pinto, A., Pereira, E., Júnior, G. B., and Rivero, L. (2019). Educação tutorial em computação: Uma análise retrospectiva das atividades realizadas para a formação pessoal e profissional de cientistas da computação. In Anais do XXVII Workshop sobre Educação em Computação, pages 101-110. SBC.

Matturro, G., Raschetti, F., and Fontán, C. (2019). A systematic mapping study on soft skills in software engineering. J. UCS, 25(1):16-41.

Pedrosa, N. and Ferreira da Silva, L. (2019). A importância dos soft skills nas descrições das vagas de gerente de projetos de ti. Revista Alcance, 26(1):45-60.

Schulz, B. (2008). The importance of soft skills: Education beyond academic knowledge. Journal of Language and Communication, pages 146-154.

Succi, C. and Canovi, M. (2020). Soft skills to enhance graduate employability: comparing students and employers' perceptions. Studies in Higher Education, 45(9):1834 1847. 